\title{
BMJ Open SLEEP: intervention with weighted blankets for children with attention deficit hyperactivity disorder (ADHD) and sleep problems: study protocol for a randomised control trial
}

\author{
Ingrid Larsson (D) , ${ }^{1,2,3}$ Katarina Aili (D) , Jens M Nygren (D) , ${ }^{1}$ Pia Johansson (D) , \\ Håkan Jarbin (D) , ${ }^{4,5}$ Petra Svedberg (D) ${ }^{1}$
}

To cite: Larsson I, Aili K, Nygren JM, et al. SLEEP: intervention with weighted blankets for children with attention deficit hyperactivity disorder (ADHD) and sleep problems: study protocol for a randomised control trial. BMJ Open 2022;12:e047509. doi:10.1136/ bmjopen-2020-047509

- Prepublication history for this paper is available online. To view these files, please visit the journal online (http://dx.doi. org/10.1136/bmjopen-2020047509).

Received 02 December 2020 Accepted 03 December 2021

Check for updates

(c) Author(s) (or their employer(s)) 2022. Re-use permitted under CC BY-NC. No commercial re-use. See rights and permissions. Published by BMJ.

${ }^{1}$ School of Health and Welfare, Halmstad University, Halmstad, Sweden

${ }^{2}$ Spenshult Research and Development Centre, Halmstad, Sweden

${ }^{3}$ Department of Clinical

Sciences, Lund University, Lund, Sweden

${ }^{4}$ Faculty of Medicine, Lund University, Lund, Sweden

${ }^{5}$ Child and Adolescent

Psychiatry, Region Halland, Halmstad, Sweden

Correspondence to Dr Ingrid Larsson; ingrid.larsson@hh.se

\section{ABSTRACT}

Introduction and objectives Children with attention deficit hyperactivity disorder (ADHD) have an increased risk of sleep problems. Weighted blankets are one possible non-pharmacological intervention for these problems in this group of children. However, the effectiveness of weighted blankets is insufficiently investigated. This study aims to investigate the effectiveness of weighted blankets in terms of sleep, health-related outcomes and cost-effectiveness as well as to explore children's and parents' experiences of a sleep intervention with weighted blankets.

Methods and analysis This study is a randomised placebo-controlled crossover trial comparing the effect of weighted fibre blankets (active) with fibre blankets without weight (control). Children aged 6-13 years, recently diagnosed with uncomplicated ADHD with verified sleep problems, were included in the study. The study period is 4 weeks for each condition, respectively, and then an 8-week follow-up. A total of 100 children diagnosed with $A D H D$ and sleep problems will enter the study. The primary outcomes are sleep and cost per quality-adjusted life years. The secondary outcomes are health-related quality of life, ADHD symptoms, psychological distress and anxiety. Interviews with a subsample of the participating children and parents will be conducted for exploring the experiences of the intervention.

Ethics and dissemination Ethical approval of the trial has been obtained from the Swedish Ethical Review Authority (number 2019--2158) and conforms to the principles outlined in the Declaration of Helsinki (WMA, 2013). Results will be reported as presentations at peerreview conferences, in articles in peer-review journals and meetings with healthcare providers.

Trial registration number NCT04180189.

\section{INTRODUCTION}

Children with attention deficit hyperactivity disorder (ADHD) have an increased risk of poor health outcomes compared with healthy children. ${ }^{12}$ The increased risk is especially apparent among girls. ${ }^{3}$ Between $25 \%$
Strength and limitations of this study

- The scientific evidence on the effectiveness of weighted blankets is insufficient.

- The results from this randomised controlled trial will provide new evidence of the efficacy, costeffectiveness and experiences of the intervention.

- The design used to evaluate the intervention of weighted blankets in the trial may be applied to other healthcare settings and may lead to the development of systematic evaluations of the intervention in local contexts.

- Weighted blankets are prescribed to patients in healthcare as a non-pharmacological intervention for sleep problems, the results from this study make it also applicable to other categories of patients than children with attention deficit hyperactivity disorder.

- Potential limitations include loss to follow-up during the multiphase study and that the trial is only implemented at one Department of Child and Adolescent Psychiatry in the southern part of Sweden, which may limit generalisability of specific study findings to other populations and settings.

and $50 \%$ of children with ADHD have sleep problems, ${ }^{12}$ commonly including bedtime resistance, night and early morning awakening and co-sleeping. ${ }^{4}$ Sleep is important for everyday functioning and essential for health and well-being. ${ }^{5-7}$ Sleep deprivation is associated with reduced quality of life, ${ }^{8}$ an increased risk of various physical and mental health consequences ${ }^{9} 10$ as well as increased risk-taking behaviour. ${ }^{11-13}$ Furthermore, poor sleep negatively affects performance ${ }^{10}$ and relationships in school, ${ }^{14}$ which has consequences for school results and transition into adulthood and working life. ${ }^{15}{ }^{16}$ Sufficient sleep duration and quality is, on the other hand, associated with improved attention, 
behaviour and cognitive functions as well as physical and mental health. ${ }^{1718}$

The use of pharmacological treatment for sleep problems is common and has increased dramatically among children with ADHD in the last 10 years, although often with unfavourable side effects. ${ }^{1} 1119$ There is evidence supporting the commonly used melatonin compared with placebo, but the degree of benefit is uncertain. ${ }^{20}$ There are various types of non-pharmacological interventions for children with ADHD to manage sleep problems, which are not associated with the side effects associated with pharmacological treatment. However, due to clinical heterogeneity, poor study quality and lack of randomised controlled trials (RCTs), ${ }^{20-23}$ the evidence for the effectiveness of non-pharmacological interventions is inconclusive. Thus, there is a need for high-quality studies to evaluate the clinical effect and cost-effectiveness of nonpharmacological sleep interventions.

Weighted blankets were being prescribed in Sweden as a supplement to or replacement of pharmacological treatment for sleep problems among children with ADHD. However, the practice was recently stopped due to lack of evidence supporting the practice. The effectiveness of weighted blankets in this context has received little research attention and has generally had insufficient scientific quality, ${ }^{21} 24$ with only one RCT. ${ }^{26}$ This latter study had a randomised, placebo-controlled crossover design with a 4-week follow-up for each type of blanket. The population consisted of 67 children, aged 5-16 years, with autism spectrum disorders. Weighted blankets, compared with the control blanket, did not increase TST, sleep-onset latency or sleep efficiency (SE) as measured by actigraphy. However, parents and children preferred the weighted blanket, and the weighted blankets were well tolerated. ${ }^{26}$ In a pilot study including only two children with an autism spectrum disorder, the use of weighted blankets improved the sleep quality, justifying the need for additional robust research. ${ }^{27}$ A case-control study without randomisation included 21 children, aged 8-13 years, with ADHD and 21 matched healthy children as a control group showed some small positive effects of the weighted blankets on sleep onset latency (SOL). ${ }^{28}$ In summary, these studies do not provide conclusive evidence of the effect, ${ }^{25}$ economic effectivess ${ }^{2021}$ or children's and parents' experiences of weighted blankets for children with ADHD on sleep problems. Thus, an RCT evaluating weighted blankets for children with ADHD and sleep problems is timely. Given the societal cost and the quality of life implications of sleep interventions, the cost-effectiveness of this non-pharmacological intervention also needs to be investigated. ${ }^{20}$ The hypothesis of this RCT is that weighted blankets will improve objectively measured and self-reported sleep compared with control blankets in children with ADHD.

\section{Objectives}

This study targets children with newly diagnosed ADHD and sleep problems and aims to (1) evaluate the effect of an intervention with weighted blankets on sleep and other health-related outcomes, (2) evaluate the costeffectiveness of weighted blankets and (3) explore children's and parents' experiences of the sleep intervention with weighted blankets.

\section{METHODS \\ Study design}

This is a randomised, placebo-controlled crossover trial investigating the effects of an intervention with fibreweighted blankets in children with ADHD. The study period is 4 months, including $2 \times 4$ weeks of intervention with weighted blankets and control blankets, followed by an 8 -week follow-up (figure 1). The study investigates the effect of the intervention in terms of (1) sleep and health; (2) cost-effectiveness and (3) experiences of sleep and health-related outcomes. The qualitative part will be performed using an explorative design based on interviews from a subsample of the included children and their parents. The interviews will be conducted at the end of the 4-month intervention in order to gain knowledge of the children's and parents' experiences of using these fibre-weighted blankets. The protocol is based on the Standard Protocol Items for Randomized Trials. ${ }^{29}$

\section{Patient and public involvement}

The design of the study and the preparation and formulation of this protocol have been coproduced with healthcare professionals at the child and adolescent mental health service (CAMHS). This includes being involved in; planning inclusion and exclusion criteria for the informants, preparing the arrangement for the intervention and selecting which questionnaires to be used to measure outcome variables for children and parents. The project manager has had regular meetings with the healthcare professionals at CAMHS throughout the preparation of the study. A pilot study, with seven children and their parents, has been performed to validate the design, interventions and questionnaires used. As part of this, we asked children and parents about their opinion of the intervention and also asked them for suggestions for improvements. This resulted in a few minor adjustments. The research project has been discussed with occupational therapists (who prescribe weighted blankets in Sweden) and representatives from the national occupational therapy association. The project and preliminary results have been and will be communicated through popular science reports, research conference contributions and research papers to the healthcare services, occupational therapists, patient groups and researchers during the research period.

\section{Participants and recruitment}

Children attending the ADHD unit at CAMHS in a county council in the southern part of Sweden during 2020-2021 will be asked to participate in the study. This ADHD unit is designed to assess and if indicated initiate treatment of 


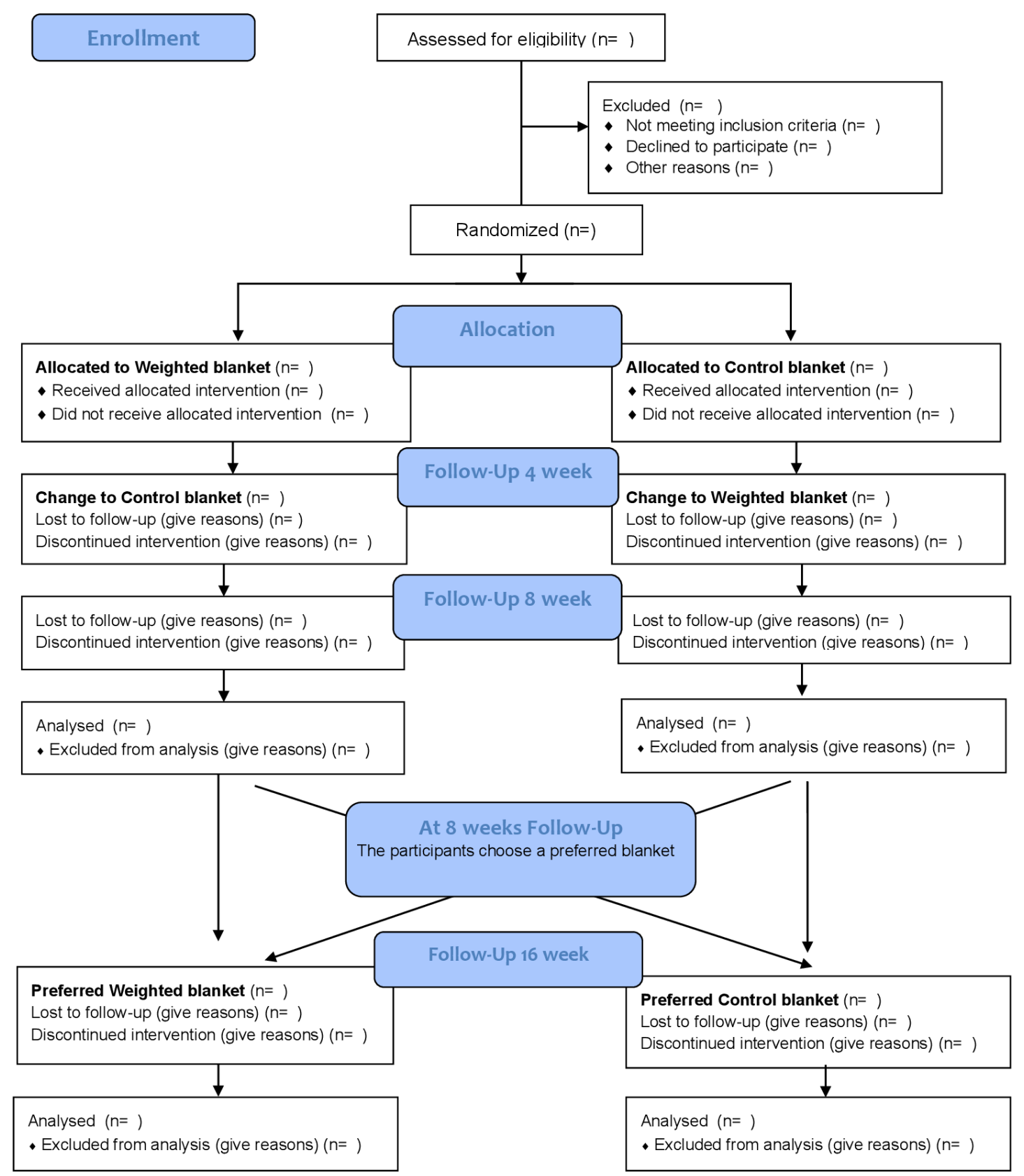

Figure 1 Flowchart RCT. The time between requirement, randomisation, intervention and follow-up. RCT, randomised controlled trial.

children with uncomplicated ADHD according to DSM$5^{30}$ in order to increase effectiveness and reduce waiting lists. About one half of patients with newly diagnosed ADHD are seen in this unit. The triaging unit selected patients ages 6-12 when the structured Brief Child and Family Phone Interview (BCFPI) suggested a probable diagnosis of ADHD. Exclusion criteria for referral to this unit were significant comorbidity requiring immediate treatment, severe parental stress and intellectual impairment requiring more comprehensive interventions. The diagnostic assessment was based on written information from the present school (teacher report form and open questions about school functioning), the BCFPI, interview with parent and the child and observing the child during the 2-hour assessment. The diagnostic schedule was inspired by a short form of Kiddie Schedule for Affective Disorders and schizophrenia-Present and Lifetime version to cover $\mathrm{ADHD}$, externalising and tic disorders as well as anxiety and affective disorders while infrequent diagnoses were not screened. ${ }^{31}$ Patients in the ADHD unit received psychoeducation in groups and medication with fewer follow-ups than usual, and insufficient for the more complicated cases. ${ }^{32}$ Recruitment of participants started in January 2020 and is estimated to be completed during autumn 2021. All children aged 6-13 years, recently diagnosed with uncomplicated ADHD with sleep problems verified by three selected questions from the Child's Sleep Habits Questionnaire (CSHQ),${ }^{33}$ will be 
approached for participation in the study. Sleep problem is considered to be present if the child (1) seldom (0-1 times per week) or sometimes (2-4 times per week) fall asleep within $20 \mathrm{~min}$ after going to bed, (2) usually (5-7 times per week) or sometimes (2-4 times per week) sleep too little or (3) wake up several times per night. In addition to this, they need to report that the sleep difficulty in question is a problem. In addition, parents and children should understand (written and spoken) the Swedish language. Children will be excluded if they have already used weighted blankets as a sleep intervention, or if they were currently on and wished to stay on melatonin for sleep problems. Children diagnosed with ADHD and not started on a stimulant and with sleep difficulties above the threshold will be invited to get further information about the study at the 3-hour assessment visit. Children diagnosed with ADHD and started on a stimulant will again be reviewed for inclusion criteria at the medication follow-up after about 4 weeks.

First, eligible children and their parents will be informed verbally about the study by their doctor or nurse at the CAMHS. They will then receive written information about the study and be approached by healthcare professionals about participation. After the researchers have received written consent, the participants will be contacted by telephone and the research project leader will provide more detailed information about the study. The participants will be informed that they are about to try two different types of fibre blankets. The participants will be encouraged to contact the researchers if further questions arise.

\section{Intervention}

The participants $(\mathrm{n}=100)$ will be randomly assigned into two groups using simple randomisation with stratification. ${ }^{34}$ A total of 100 sheets with the letter A (intervention) or the letter B (control) will be printed out and put into each envelope. The sealed envelopes will be mixed and one of the researchers will pick an envelope randomly for each child. The letter A or B indicates if the child will start with a fibre-weighted blanket (active) or with a fibre blanket without weight (control). After 4 weeks with either the active intervention (A) or control (B), the children will change blanket (if starting with active, the child will change into control, and vice versa). After this 8-week period, the child will decide which of the two blankets (active or control) that they want to retain.

Fibre-weighted blankets from Novista of Sweden ( Novista.se) will be used in this study. The weight in the blankets is derived from longitudinal polyester fibres permitting flexibility. The blanket size is $150 \times 210 \mathrm{~cm}$, which is a standard size for children and adults in Sweden. The weight of each blanket will be individually tailored (weight between $6 \mathrm{~kg}$ and $10 \mathrm{~kg}$ ) for the children, based on age, sex, height, weight, degree of sleep problems and subtypes of ADHD of two independent experienced occupational therapists. The fibre blankets without weight (controls) have been designed for the project, so that both active and control blankets have the same design. The weight is the only aspect that distinguishes them.

\section{Data assessment}

Data will be assessed at baseline (prior to the intervention) and during the 4th, 8th and 16th weeks of the study. The measurements are performed during the last of the 4 weeks to minimise the risk of bias due to carry over effects. A 7-day objective measurement of sleep will be conducted during these measurement periods. Self-reported data will also be gathered through the completion of a questionnaire by the parent and child, respectively (table 1 ).

Table 1 Overview of the questionnaires included in the study

\begin{tabular}{|c|c|c|}
\hline & $\begin{array}{l}\text { Assessed by children } \\
\text { reports }\end{array}$ & Assessed by parents' reports \\
\hline Socioeconomic variables & & $\begin{array}{l}\text { Children: age, gender, country of birth, } \\
\text { Parents: age gender, country of birth, civil status, level of education, and work } \\
\text { situation }\end{array}$ \\
\hline Sleep habits & Insomnia Severity Index ${ }^{39}$ & Child's Sleep Habits Questionnaire ${ }^{33}$ \\
\hline General well-being & $\begin{array}{l}\text { Children Outcome Rating } \\
\text { Scale }^{40}\end{array}$ & Outcome Rating Scale 4047 \\
\hline
\end{tabular}

\section{ADHD symptoms}

Family situation and parental mood

Health-related quality of life EQ-5D-Y $Y^{5153}$

Resource consumption
Swanson, Peland and Nolan Scale 4445

The Brief Child and Family Phone Interview ${ }^{49} 50$

EQ-5D-3L 5257

School absence (children), work productivity and absence (parents), and healthcare consumption according to Swedish adaptation ${ }^{5455}$ of the TIC-P instrument $^{56}$

ADHD, attention deficit hyperactivity disorder; EQ-5D-3L, EuroQoL 5-Dimension 3-Level; EQ-5D-Y, EuroQol 5-Dimension Youth; TIC-P, Treatment Inventory of Costs in Patients with psychiatric disorders . 
Interviews with an adequate sample of children $(n=25)$ and parents $(n=25)$ will be conducted after the intervention period in order to understand the experiences of the intervention's impact on sleep and health-related outcome.

\section{Methods for investigating the health effects of weighted blankets \\ Primary outcome}

The primary outcome is objectively measured and self-reported sleep. Variables of interest from the objectively measured sleep are: Sleep onset latency (SOL), which refers to the period of time between turning lights out to go to sleep (timing identified by marker from the event button or selfreported time in daily text messages) and falling asleep; total sleep time (TST), which is equal to the time of total sleep episode minus the awake time (the entire time spent sleeping); sleep efficiency (SE), which is the actual sleep time expressed as a percentage of the total time in bed (the time elapsed between "lights out" and "get up time)"; wake after sleep onset (WASO), referring to periods of wakefulness occurring after sleep onset.

Objectively measured sleep will be assessed using actigraphy. This method for assessing sleep has been shown to be valid in several studies ${ }^{35}$ and has shown to be strongly associated with polysomnographic measures with a correlation coefficient of at least 0.85 in healthy individuals. ${ }^{36}$ Measurements from at least 4-7 nights have been recommended. ${ }^{37}$ Motionwatch V.8 (Camntech), a triaxial accelerometer using Micro-electromechanical systems (MEMs) technology, capable of sensing motions in a resultant force range of $0.01-8 \mathrm{~g}^{38}$ is used in this study. The actigraph registers total gross motor activity for analysis of sleep-wake patterns and has good validity for measuring sleep. ${ }^{38}$ Recordings will be taken in 30 s epochs.

Participants will be instructed to wear the watch on their non-dominant wrist, for seven consecutive nights. If not worn during the day, the parent and child will be instructed to put on the watch in the early evening, or in good time prior to going to bed. The participant is instructed to push an event-button when they decide to go to sleep, for example, when they stop reading a book or turn off the lights. In addition to marking the event of going to sleep and waking up by pressing the button, the parents will answer questions daily (by text message): (1) What time did your child go to bed yesterday?; (2) How long time do you estimate the time for your child to fall asleep from the time your child went to bed? (hours, min); (3) What time did your child wake up today?; (4) Was your child restless and moved around a lot during sleep? (not at all restless, a little restless, moderately restless, very restless); (5) Was your child restless when falling asleep? (not at all restless, a little restless, moderately restless, very restless)

The variables of interest from the self-reported sleep will be assessed by $\mathrm{CSHQ}^{33}$ and Insomnia Severity Index (ISI) ${ }^{39}$

The CSHQ assesses parental reported sleep and consists of 33 items related to eight subscales; (1) Bedtime resistance,
(2) Sleep onset delay, (3) Sleep duration, (4) Sleep anxiety, (5) Night wakings, (6) Parasomnias, (7) Sleep-disordered breathing and (8) Daytime sleepiness. Each item is rated on a threepoint scale: 'usually' if the sleep behaviour occurred five to seven times/week; 'sometimes' for two to four times/ week and 'rarely' for zero to one time/week. A higher score indicates more sleep problems. The scale has good reliability and validity. ${ }^{33}$

Insomnia will be assessed by ISI, which comprises seven items for the children to respond to: (1) Severity of sleep onset, (2) Sleep maintenance, (3) Early morning awakening, (4) Satisfaction with current sleep pattern, (5) Interference with daily functioning, (6) Noticeability of impairment attributed to the sleep problem and (7) Level of distress caused by the sleep problem. Each item is rated on a 5-point Likert scale ranging from 'not at all' (scored at 0 ) to 'extremely' (scored at 4). Total score ranges from 0 to 28, with higher scores indicating greater severity. ISI is a reliable and valid instrument for quantifying the severity of perceived insomnia and measures insomnia in treatment research. ${ }^{39}$ The ISI will be slightly modified in order to better correspond to a child's language.

\section{Secondary outcomes}

The children's general well-being will be assessed by the Child Outcome Rating Scale (CORS), ${ }^{40}$ which is an overall measure of psychological distress. It was developed to give children a voice in the services they receive. CORS comprises four items where the child evaluates (1) Me (How am I doing?), (2) Family (How are things in my family?), (3) School (How am I doing at school?) and (4) Everything (How is everything going?). Each item is rated on a 100-millimeter Visual Analog Scale with smiling and sad faces as anchors. CORS has good reliability and moderate validity. ${ }^{40}$

The children's anxiety will be assessed by The short StateTrait Anxiety Inventory for children (short-STAI). ${ }^{41}{ }^{42}$ Short-STAI includes six items. ${ }^{43}$ Each item is rated on a 4-point Likert scale ranging with $1=$ 'not at all', 2='somewhat', $3=$ ='moderately' and $4=$ "very much'. The total score range from 6 to 24 points, with six points indicating no anxiety and 24 points indicating the highest level of anxiety. Short-STAI has good reliability and validity for children. ${ }^{41}$

The children's ADHD symptoms-hyperactivity/impulsivity, inattention and oppositional-will be assessed by the parents filling in The Swanson, Peland and Nolan Scale (SNAP-IV). ${ }^{44}$ The SNAP-IV consists of 30 items and is divided into three subscales: inattention (nine items), hyperactivity/impulsivity (nine items) and oppositionality (eight items) and four supplementary questions regarding oppositionality (two questions) and ADHD (two questions). Items are rated on a 4-point Likert scale range $0=$ 'not at all', $1=$ 'just a little', $2=$ 'quite a bit' and $3=$ 'very much'. Items for inattention and hyperactivity/ impulsivity can be combined to create a 'combined ADHD' score. ${ }^{45}$ Higher scores represent more symptoms. 
The SNAP-IV is a robust and valid measure of outcome for research studies and is often used in RCTs. ${ }^{46}$

The parents' general well-being will be assessed by the Outcome Rating Scale (ORS) ${ }^{47}$ which is a general mental health assessment of the past week in four items; (1) Personal well-being, (2) Interpersonal relationships, (3) Social relations and (4) Overall sense of well-being. Each item is rated on a 100-millimeter Visual Analog Scale with anchors from 0 (negative) to 100 (positive). ORS is a reliable and valid instrument. ${ }^{40} 48$

Family situation and parental mood will be assessed by the BCFPI ${ }^{49}{ }^{50}$ BCFPI is a structured parent interview for triage at intake and follow-up evaluation of community care at CAMHS. It consists of 36 symptom items and another 36 items to assess function, adversity and family stress grouped into 12 subscales. The subscale 'family situation' contains three items rated on a 4-point Likert scale range $1=$ never, $2=$ sometimes, $3=$ often and $4=$ always. The subscale 'parental mood' contains six items based on the question 'How often during the past week has the parent experienced...?' rated on a 4-point scale; <1 day, 1-2 days, $3-4$ days and $>5$ days. BCFPI has good reliability and validity. ${ }^{49} 50$

Health-related quality of life will be assessed for children with EuroQol 5-Dimension Youth (EQ-5D-Y) ${ }^{51}$ and parents with EuroQoL 5-Dimension 3-Level (EQ-5D-3L). ${ }^{52}$ EQ-5D-Y measures health-related quality of life 'today' for children and young people and is developed from the standard adult EQ-5D. ${ }^{51}$ EQ-5D-Y comprises five items: (1) Walking about (mobility), (2) Looking after myself (self-care), (3) Doing usual activities (usual activities), (4) Having pain or discomfort (pain and discomfort) and (5) Feeling worried, sad or unhappy (anxiety and depression). Each item is divided into three levels: No problems, Some problems and A lot of problems. The EQ-5D-Y also includes an easily understandable modified vertical Visual Analogue Scale of EQ-5D, where the respondent rates the overall health status with the endpoints from 0 (the worst health state the child can imagine) to 100 (the best health state the child can imagine).$^{51}$ EQ-5D-Y has good reliability and validity. ${ }^{53}$

EQ-5D is a generic health-related quality of life instrument ${ }^{52}$ measuring the parents' health comprising five dimensions: (1) Mobility, (2) Self-care, (3) Usual activities, (4) Pain/discomfort and (5) Anxiety/Depression. Each dimension is divided into three levels: no problems, some or moderate problems and extreme problems. In addition to the five dimensions, a 100-millimeter vertical Visual Analog Scale with endpoints of 100 means 'best imaginable health state' and 0 means 'worst imaginable health state is included'. The total score ranges from 0 to 1 , where a higher score indicates a better health-related quality of life.

ADHD diagnosis and subtype as well as comorbidities will be extracted from the clinical records. In addition, symptom load from ADHD inattention or/and hyperactivity/impulsivity pharmacological treatment, sociodemographic data, resource consumption and will be collected via the survey at baseline and the 4 th, 8 th and 16 th weeks of the study.

\section{Methods for investigating the cost-effectiveness of weighted blankets}

The health economic evaluation is a within-trial costutility analysis with a societal perspective based on the data collected at the 8-week follow-up, with the primary outcome costs per quality-adjusted life years (QALY). An incremental cost-effectiveness ratio (ICER) with a 4-week time horizon is calculated based on differences in societal costs (implementation costs and societal consequences) and quality of life when using either the fibre-weighted blankets or control blankets. No discounting of costs and health effects will be performed due to the short follow-up period. A number of sensitivity analyses are planned, including probabilistic analyses with bootstrapped differences of individual-level data on major societal consequences and quality of life as well as of the ICER.

The societal cost consequences combine parent-reported data on resource consumption with clinical register data to estimate the differences in societal costs between the two study arms at baseline and 8 weeks. The parentreported resource consumption survey questions are based on a Swedish adaptation of the Treatment Inventory of Costs in Patients with psychiatric disorders (TIC-P) instrument ${ }^{54-56}$ and consider the 4 weeks. The questions include school absence for the child (in numbers of full days, half days and 1-2 hours), work absence for one parent (in numbers of full days (8 hours), three-quarters of a day (6 hours), half a day (4 hours) and one-quarter of a day (2hours)), work productivity of the parent (in 10 levels from no work accomplished to hardly no decreased work capacity) and healthcare appointments (nine types of healthcare including, eg, school healthcare, primary care and emergency care). The clinical register data include the number of appointments at the CAMHS and prescribed pharmacological therapy. The cost of the resource consumption items from the parent survey and the clinical register will be estimated according to Swedish published data on the average costs for healthcare appointments from the Swedish Association of Local Authorities and Regions (SALAR) and occupationspecific healthcare wages including wage taxes from Statistics Sweden. The cost of parents' work absence and decreased productivity will be estimated using average Swedish hourly wages including wage taxes from Statistics Sweden while the cost of child school absence is estimated according to estimated schooling costs from The Swedish National Agency for Education. The cost of prescribed pharmacological therapy will be estimated according to listed prices at the Dental and Pharmaceutical Benefits Agency.

The implementation costs, that is, the prescription of the weighted blankets, include the healthcare region's purchasing price for weighted blankets, administration and transportation from the assistive technology centre, child psychiatrist time for referral to an occupational 
therapist, occupational therapist time for assessment, prescription and tailoring as well as parent and child time. The healthcare costs will be estimated based on data from SALAR and Statistics Sweden while the visiting and travelling time for the parent and child will be estimated according to data from Statistics Sweden and The Swedish National Agency for Education, as above.

Quality of life estimates for calculating QALYs over the 4 weeks period will be taken from the parent-reported EQ-5D instrument valued with a Swedish tariffs value set. ${ }^{57}$ EQ-5D-3L is frequently used in Sweden and for economic evaluations and is considered a reliable and valid instrument. ${ }^{57}$ There is currently no appropriate value set for the child version of EQ-5D (ie, EQ-5D-Y), so in a sensitivity analysis, the VAS ratings will be used and added to the parent-estimated QALYs. The QALYs during 4 weeks will be calculated based on the mean changes in quality of life of using weighted blankets versus control blankets, with an instant change assumed when initiating use of the weighted blankets.

\section{Methods for investigating the experiences of weighted blankets}

The qualitative data will consist of individual interviews with children and their parents in the intervention study. An open interview guide with initial questions will be used to ensure similar data from all participants. The initial questions refer to the experiences of sleep for children with ADHD, experiences of how the sleep intervention with weighted blankets influences the children's sleep and health-related outcomes as well as the family situation. Questions to the children: 'How do you usually sleep?', 'In what way can it be difficult to sleep?', 'How does sleep differ if you sleep well or badly?', 'What is important for you to be able to sleep?', 'How do you experience the two different blankets you have used?', 'How do you experience the weighted blanket?', 'Can you describe your sleep since you started using the weighted blankets?'. Questions to the parents: 'What does sleep mean for your child?', 'How does your child usually sleep?', 'What is important for your child to be able to sleep?', 'How is your child's life affected by sleep?', 'How do you experience the two different blankets your child has used?', 'How do you experience the weighted blanket?', 'Can you describe your child's sleep since he/she started using the weighted blankets?', 'How is your child's well-being since he/she started using the weighted blankets?', 'How has the situation for the family and you as a parent been affected since your child started using the weighted blanket?' Follow-up probes will be used to encourage children and parents to elaborate on the answers: 'Please tell me more' 'How do you mean?' or 'What do you have in mind when you say...?' The interviews will be digitally recorded and transcribed verbatim.

\section{Statistical power}

A power analysis was made based on estimated changes in the primary outcome variable SOL. Estimations of mean and SD were made based on previous studies of SOL in children. Mean SOL is expected to differ substantially with age among children. Gringras et al investigated children $5-16$ years of age and found a mean value of $76,5 \mathrm{~min}$ of SOL with an SD of 46.1. ${ }^{26}$ Hvolby and Bilenberg studied SOL in children 8-13 years of age and reported a mean value of $23.1 \mathrm{~min}$ of SOL with an SD of $9.4 .^{28}$

Previous studies investigating the effect of similar interventions on SOL have found a $40 \%$ decrease in SOL after the intervention. ${ }^{28}$ The power calculation of this study is based on the assumption that a $30 \%$ decrease in SOL is a clinically relevant improvement. In this study, including children 6-13 years of age, estimating a mean of $35 \mathrm{~min}$ SOL and an SD of 15 , the power analysis demonstrated that 58 children (29 in each group) will be a sufficient number if accepting a $30 \%$ difference between groups in SOL with $80 \%$ power. To allow for a $40 \%$ dropout, 100 children (50 in each group) will be enrolled in the study.

\section{Data analysis}

Statistical analyses will be performed using SPSS V.24 for Windows. The intervention will be evaluated in terms of effect and cost comparison. Differences will be evaluated with an intention to treat analysis. Objectively and subjectively measured sleep, anxiety and health-related quality of life will be evaluated with a paired t test or equivalent non-parametric tests and by independent sample $t$ test for between-group analyses of carry over and period effect. Children included are stable on medication before inclusion and are encouraged not to initiate other sleep adjustments during the study period. ADHD symptoms and sleep problems are, thus, considered to be stable over the $4+4$ cross over period minimising any period effects. The weighted blanket is only active under actual use, and the treatment effect is not likely to be carried over. Linear mixed-effect model will be used for evaluating the effect on sleep problems over time. Differences in societal costs will be analysed via non-parametric bootstrap analyses on individual-level data and reported as credibility intervals. ${ }^{58}$ The qualitative data from interviews will be analysed with inductive qualitative content analysis. ${ }^{59}$

\section{ETHICS AND DISSEMINATION}

The study is approved by the Swedish Ethical Review Authority (number 2019-02158) and conforms to the principles outlined in the Declaration of Helsinki. ${ }^{60}$ The study will fulfil the requirements for research: information, consent, confidentiality and safety of the participants and is guided by the ethical principles: autonomy, beneficence, non-maleficence and justice. ${ }^{61}$ All participation and data collection will be performed confidentially. Children and parents will receive written and oral information and parents give their informed consent in writing. The participants will be informed that they can withdraw from the project at any time without having to justify why. Data will be collected in anonymised form and keys that link data with personal information will 
be stored separately and only accessible to the project leader. All personal data will be registered according to the General Data Protection Regulation (2016/679), ${ }^{62}$ and the data will be stored in accordance with the Archive Act in Sweden (SFS1990:782).$^{63}$ This study is registered at http://clinicaltrials.gov. The results of this study will be communicated to the included participants, healthcare providers and companies, in manuscripts submitted to peer-reviewed journals as well as in presentations at national and international peer-review conferences.

\section{DISCUSSION}

Weighted blankets are prescribed to patients in healthcare in Sweden and are widely used as a non-pharmacological intervention for sleep problems, even though evidence for the effects of weighted blankets is lacking. The few previous studies investigating the effect of weighted blankets for children with ADHD have not shown conclusive results and, to date, there are too few high-quality studies to support the intervention. ${ }^{25-28}$ The results from this RCT will, thus, be important for providing new evidence of the efficacy, cost-effectiveness and experiences of the use of weighted blankets to address sleep problems among children with ADHD.

Some methodological considerations can be highlighted. A strength is that this study will be the first randomised placebo-controlled crossover trial investigating the effects of fibre-weighted blankets in children with ADHD. Another strength is the use of both objective and subjective measures for sleep. Although subjectively measured sleep is highly relevant to assess and evaluate, objectively measured sleep has the advantages of being free from subjective expectations in relation to the intervention and less sensitive to recall bias. Furthermore, the evaluation of cost-effectiveness of weighted blankets is highly relevant though this has not previously been studied. ${ }^{21}$ The costs and benefits of the intervention need to be taken into consideration when implementing the intervention in healthcare settings. Similarly, the inclusion of a qualitative approach in the design to increase the understanding of both children's and parents' experiences of effects is another strength. This latter aspect is of great relevance as children's perspectives are seldom taken into account in research.

There are, however, a few methodological challenges with this study. Assessing self-reported data from children is difficult for several reasons. Some of the questionnaires in this study are designed for the parent to respond on behalf of the child (eg, CSHQ). This may be a good approach for younger children, but depending on the habits around bedtime, the parents may only have (at best) a reasonably good perception of how the child's sleep was (eg, if sleeping in separate bedrooms). Under these circumstances, the parent and the child are instructed to fill in the questionnaire together to get a more reliable assessment. Another potential bias is the control blankets. The difference in weight will be obvious for parents and also for children. The participants are only informed that they are trying two different kinds of blankets. However, most have learnt about weighted blankets through media or their health providers, possibly affecting expectations in favour of the weighted blanket

We anticipate that the project will make several scientific contributions to the research on health-related outcomes, sleep and cost-effectiveness for non-pharmacological sleep interventions, such as weighted blankets. These findings will be essential for healthcare professionals in their practice though evidence today for the effects of weighted blankets is scarce. The results will also be relevant for children with ADHD, in particular, but will also be relevant for other target groups and other settings.

Acknowledgements We thank the healthcare professionals at the CAMHS in Region Halland for assistance with study recruitment from their sites. We are grateful for the assistance provided by Novista of Sweden AB to provide the study with fibre blankets with and without weights.

Contributors IL, JN and PS contributed to the conception of the study, obtained the funding, and are the guarantors of the study. IL, KA, JN, PJ, HJ and PS contributed to the study design. IL and KA were primarily responsible for the statistical analysis plan. PJ was responsible for the health economic analysis plan. IL and PS were responsible for the qualitative analysis plan. IL drafted the manuscript and KA, JN, PJ, HJ and PS revised the manuscript critically for important intellectual content. All authors read and approved the final version of the manuscript.

Funding This work was supported by the Knowledge Foundation (Grant no. 20200012), Swedish Research Council for Health Working Life and Welfare (Forte), (Grant no. 2021-00664) and Region Halland (Grant no. HALLAND-940226).

Disclaimer Neither the CAMHS in Region Halland and the company Novista of Sweden AB nor the funders have any role in the study design, data collection, management, analysis, or interpretation of the data.

Competing interests None declared.

Patient and public involvement Patients and/or the public were involved in the design, or conduct, or reporting, or dissemination plans of this research. Refer to the Methods section for further details.

Patient consent for publication Consent obtained from parent(s)/guardian(s)

Provenance and peer review Not commissioned; externally peer reviewed.

Open access This is an open access article distributed in accordance with the Creative Commons Attribution Non Commercial (CC BY-NC 4.0) license, which permits others to distribute, remix, adapt, build upon this work non-commercially, and license their derivative works on different terms, provided the original work is properly cited, appropriate credit is given, any changes made indicated, and the use is non-commercial. See: http://creativecommons.org/licenses/by-nc/4.0/.

\section{ORCID iDs}

Ingrid Larsson http://orcid.org/0000-0002-4341-660X

Katarina Aili http://orcid.org/0000-0003-4260-7399

Jens M Nygren http://orcid.org/0000-0002-3576-2393

Pia Johansson http://orcid.org/0000-0002-1826-7374

Håkan Jarbin http://orcid.org/0000-0003-3533-453X

Petra Svedberg http://orcid.org/0000-0003-4438-6673

\section{REFERENCES}

1 Eyuboglu M, Eyuboglu D. Behavioural sleep problems in previously untreated children with attention deficit hyperactivity disorder. Psych Clin Psychopharmacol 2018;28:19-24.

2 Bondopadhyay U, Diaz-Orueta U, Coogan AN. A systematic review of sleep and circadian rhythms in children with attention deficit hyperactivity disorder. J Atten Disord 2022;26:149-224.

3 Becker SP, Cusick CN, Sidol CA, et al. The impact of comorbid mental health symptoms and sex on sleep functioning in children with ADHD. Eur Child Adolesc Psychiatry 2018;27:353-65. 
4 Rigney G, Ali NS, Corkum PV, et al. A systematic review to explore the feasibility of a behavioural sleep intervention for insomnia in children with neurodevelopmental disorders: a transdiagnostic approach. Sleep Med Rev 2018;41:244-54.

5 Roberts RE, Roberts CR, Duong HT. Sleepless in adolescence: prospective data on sleep deprivation, health and functioning. $J$ Adolesc 2009;32:1045-57.

6 Shochat T, Cohen-Zion M, Tzischinsky O. Functional consequences of inadequate sleep in adolescents: a systematic review. Sleep Med Rev 2014;18:75-87.

7 Bartel KA, Gradisar M, Williamson P. Protective and risk factors for adolescent sleep: a meta-analytic review. Sleep Med Rev 2015;21:72-85.

8 Amaral MOP, de Almeida Garrido AJ, de Figueiredo Pereira C, et al. Quality of life, sleepiness and depressive symptoms in adolescents with insomnia: a cross-sectional study. Aten Primaria 2017:49:35-41.

9 Medic G, Wille M, Hemels ME. Short- and long-term health consequences of sleep disruption. Nat Sci Sleep 2017;9:151-61.

10 Paruthi S, Brooks LJ, D'Ambrosio C, et al. Consensus statement of the American Academy of sleep medicine on the recommended amount of sleep for healthy children: methodology and discussion. $J$ Clin Sleep Med 2016;12:1549-61.

11 Owens J, Adolescent Sleep Working Group, Committee on Adolescence. Insufficient sleep in adolescents and young adults: an update on causes and consequences. Pediatrics 2014;134:e921-32.

12 Owens J, Wang G, Lewin D, et al. Association between short sleep duration and risk behavior factors in middle school students. Sleep 2017;40. doi:10.1093/sleep/zsw004. [Epub ahead of print: 01 Jan 2017].

13 Telzer EH, Fuligni AJ, Lieberman MD, et al. The effects of poor quality sleep on brain function and risk taking in adolescence. Neuroimage 2013;71:275-83.

14 Holdaway AS, Becker SP. Children's sleep problems are associated with poorer student-teacher relationship quality. Sleep Med 2018;47:100-5.

15 Singh K, Zimmerman AW. Sleep in autism spectrum disorder and attention deficit hyperactivity disorder. Semin Pediatr Neurol 2015;22:113-25.

16 Thapar A, Cooper M. Attention deficit hyperactivity disorder. Lancet 2016;387:1240-50.

17 de Bruin EJ, van Run C, Staaks J, et al. Effects of sleep manipulation on cognitive functioning of adolescents: a systematic review. Sleep Med Rev 2017;32:45-57.

18 Reddy R, Palmer CA, Jackson C, et al. Impact of sleep restriction versus idealized sleep on emotional experience, reactivity and regulation in healthy adolescents. J Sleep Res 2017;26:516-25.

19 Esposito S, Laino D, D'Alonzo R, et al. Pediatric sleep disturbances and treatment with melatonin. J Trans/ Med 2019;17:77.

20 Beresford B, McDaid C, Parker A, et al. Pharmacological and nonpharmacological interventions for non-respiratory sleep disturbance in children with neurodisabilities: a systematic review. Health Technol Assess 2018;22:1-296.

21 Scantlebury A, Mcdaid C, Dawson V, et al. Non-pharmacological interventions for non-respiratory sleep disturbance in children with neurodisabilities: a systematic review. Dev Med Child Neurol 2018;60:1076-92.

22 Bourchtein E, Langberg JM, Eadeh H-M. A review of pediatric nonpharmacological sleep interventions: effects on sleep, secondary outcomes, and populations with co-occurring mental health conditions. Behav Ther 2020;51:27-41.

23 Busch V, Altenburg TM, Harmsen IA, et al. Interventions that stimulate healthy sleep in school-aged children: a systematic literature review. Eur J Public Health 2017;27:53-65.

24 France KG, McLay LK, Hunter JE, et al. Empirical research evaluating the effects of non-traditional approaches to enhancing sleep in typical and clinical children and young people. Sleep Med Rev 2018;39:69-81.

25 Eron K, Kohnert L, Watters A, et al. Weighted blanket use: a systematic review. Am J Occup Ther 2020;74:7402205010p1-7402205010.

26 Gringras P, Green D, Wright B, et al. Weighted blankets and sleep in autistic children--a randomized controlled trial. Pediatrics 2014;134:298-306

27 Gee BM, Peterson TG, Buck A, et al. Improving sleep quality using weighted blankets among young children with an autism spectrum disorder. Int J Ther Rehabil 2016;23:173-81.

28 Hvolby A, Bilenberg N. Use of ball blanket in attention-deficit/ hyperactivity disorder sleeping problems. Nord J Psychiatry 2011;65:89-94.
29 Chan A-W, Tetzlaff JM, Gøtzsche PC, et al. SPIRIT 2013 explanation and elaboration: guidance for protocols of clinical trials. BMJ 2013;346:e7586.

30 American Psychiatric Association. Diagnostic and statistical manual of mental disorders (DSM-5). Washington, DC: American Psychiatric Association Publishing, 2013

31 Jarbin $\mathrm{H}$, Andersson M, Råstam M, et al. Predictive validity of the $\mathrm{K}$ SADS-PL 2009 version in school-aged and adolescent outpatients. Nord J Psychiatry 2017;71:270-6.

32 Wernersson R, Johansson J, Andersson M, et al. Evaluation of a new model for assessment and treatment of uncomplicated ADHD - effect, patient satisfaction and costs. Nord J Psychiatry 2020;74:96-104.

33 Owens JA, Spirito A, McGuinn M. The children's sleep habits questionnaire (CSHQ): psychometric properties of a survey instrument for school-aged children. Sleep 2000;23:1-9.

$34 \mathrm{Kim}$ J, Shin W. How to do random allocation (randomization). Clin Orthop Surg 2014;6:103-9.

35 Sadeh A. The role and validity of actigraphy in sleep medicine: an update. Sleep Med Rev 2011;15:259-67.

36 Sadeh A, Hauri PJ, Kripke DF, et al. The role of actigraphy in the evaluation of sleep disorders. Sleep 1995;18:288-302.

37 Aili K, Åström-Paulsson S, Stoetzer U, et al. Reliability of actigraphy and subjective sleep measurements in adults: the design of sleep assessments. J Clin Sleep Med 2017;13:39-47.

38 Elbaz M, Yauy K, Metlaine A. Validation of a new actigraph motion watch versus polysomnography on 70 healthy and suspected sleepdisordered subjects. J Sleep Res 2012;21:218.

39 Bastien $\mathrm{CH}$, Vallières $\mathrm{A}$, Morin $\mathrm{CM}$. Validation of the insomnia severity index as an outcome measure for insomnia research. Sleep Med 2001:2:297-307.

40 Duncans BL, Sparks JA, Miller SD. Giving youth a voice: a preliminary study of the reliability and validity of a brief outcome measure for children, adolescents, and caretakers. J Brief Ther 2006;5.

41 Nilsson S, Buchholz M, Thunberg G. Assessing children's anxiety using the modified short State-Trait anxiety inventory and talking mats: a pilot study. Nurs Res Pract 2012;2012:932570-7.

42 Spielberger CD. State-trait Anxiety Inventory for Children:(" how I Feel Questionnaire"). Consulting Psychologists Press, 1973.

43 Marteau TM, Bekker $\mathrm{H}$. The development of a six-item short-form of the state scale of the Spielberger State-Trait anxiety inventory (STAI). Br J Clin Psychol 1992;31:301-6.

44 Swanson JM, Kraemer HC, Hinshaw SP, et al. Clinical relevance of the primary findings of the MTA: success rates based on severity of $\mathrm{ADHD}$ and odd symptoms at the end of treatment. J Am Acad Child Adolesc Psychiatry 2001;40:168-79.

45 Bussing R, Fernandez M, Harwood M, et al. Parent and teacher SNAP-IV ratings of attention deficit hyperactivity disorder symptoms: psychometric properties and normative ratings from a school district sample. Assessment 2008;15:317-28.

46 Hall CL, Guo B, Valentine AZ, et al. The validity of the SNAP-IV in children displaying ADHD symptoms. Assessment 2020;27:1258-71.

47 Miller SD, Duncan B, Brown J. The outcome rating scale: a preliminary study of the reliability, validity, and feasibility of a brief visual analog measure. J Brief Ther 2003;2:91-100.

48 Campbell A, Hemsley S. Outcome rating scale and session rating scale in psychological practice: clinical utility of ultra-brief measures. Clinical Psychologist 2009;13:1-9.

49 Boyle MH, Cunningham CE, Georgiades K, et al. The brief child and family phone interview (BCFPI): 2 . usefulness in screening for child and adolescent psychopatholog. J Child Psychol Psychiatry 2009;50:424-31.

50 Cunningham CE, Boyle MH, Hong S, et al. The brief child and family phone interview (BCFPI): 1. rationale, development, and description of a computerized children's mental health intake and outcome assessment tool. J Child Psychol Psychiatry 2009;50:416-23.

51 Wille N, Badia X, Bonsel G, et al. Development of the EQ-5D-Y: a child-friendly version of the EQ-5D. Qual Life Res 2010;19:875-86.

52 Rabin R de Charro F. EQ-5D: a measure of health status from the EuroQol group. Ann Med 2001;33:337-43.

53 Ravens-Sieberer U, Wille N, Badia X, et al. Feasibility, reliability, and validity of the EQ-5D-Y: results from a multinational study. Qual Life Res 2010;19:887-97.

54 Lenhard F, Ssegonja R, Andersson E, et al. Cost-effectiveness of therapist-guided internet-delivered cognitive behaviour therapy for paediatric obsessive-compulsive disorder: results from a randomised controlled trial. BMJ Open 2017;7:e015246.

55 Sampaio F, Bonnert M, Olén O, et al. Cost-effectiveness of internetdelivered cognitive-behavioural therapy for adolescents with irritable bowel syndrome. BMJ Open 2019;9:e023881. 
56 Bouwmans C, De Jong K, Timman R, et al. Feasibility, reliability and validity of a questionnaire on healthcare consumption and productivity loss in patients with a psychiatric disorder (TiC-P). BMC Health Serv Res 2013;13:217.

57 Burström K, Sun S, Gerdtham U-G, et al. Swedish experience-based value sets for EQ-5D health states. Qual Life Res 2014;23:431-42.

58 Drummond MF, Sculpher MJ, Claxton K. Methods for the economic evaluation of health care programmes. Oxford university press, 2015.

59 Graneheim UH, Lindgren B-M, Lundman B. Methodological

challenges in qualitative content analysis: a discussion paper. Nurse Educ Today 2017;56:29-34.
60 World Medical Association. World Medical association Declaration of Helsinki: ethical principles for medical research involving human subjects. JAMA 2013;310:2191-4.

61 Sweden Research Council. Good research practice. Bromma, Sweden, 2017. Available: https://vr.se/download/18.5639980c162791bb fe697882/1523378510652/Good-Research-Practice_VR_2017.pdf

62 GDPR2016/679. General data protection regulation (GDPR) 2016/679. Brussel: European Union.

63 SFS1990:782. Archive act in Sweden Stockholm. Ministry of Culture. 